\title{
Imaging optical near-fields of nanostructures
}

\author{
P. Leiderer, ${ }^{\text {a) }}$ C. Bartels, ${ }^{\text {b) }}$ J. König-Birk, M. Mosbacher, and J. Boneberg \\ University of Konstanz, Universitätsstrasse 10, 78457 Konstanz, Germany
}

(Received 17 June 2004; accepted 16 September 2004)

\begin{abstract}
We present a method for imaging the optical near-fields of nanostructures, which is based on the local ablation of a smooth silicon substrate by means of a single, femtosecond laser pulse. At those locations, where the field enhancement due to a nanostructure is large, substrate material is removed. The resulting topography, imaged by scanning electron or atomic force microscopy, thus reflects the intensity distribution caused by the nanostructure at the substrate surface. With this method one avoids a possible distortion of the field distribution due to the presence of a probe tip, and reaches a resolution of a few nanometers. Several examples for the optical near-field patterns of dielectric and metallic nanostructures are given.
\end{abstract}

The optical properties of nanostructures are an important issue in nanoscience with a tremendous potential for applications. This holds both for individual particles and particle arrays, and for dielectric materials (e.g., photonic crystals) as well as metals (e.g., optical antennas). ${ }^{1-3}$ The optical nearfields of such particles, which are essential for understanding their function, are not easily accessible by experimental means. One approach is to use a scanning near-field optical microscope to image the intensity distribution in the vicinity of the nanostructures with a fine aperture. ${ }^{4}$ Different approaches have improved the resolution to below $25 \mathrm{~nm}$., We introduce here an alternative method that consists in imaging optical near-field intensities by means of intense short laser pulses. This technique also reaches a resolution of a few nanometers, much smaller than the laser wavelength used, but which, moreover, is not hampered by possible distortions of the resulting patterns due to the presence of a probe tip.

In our experiments the nanoparticles, located on a smooth substrate, are irradiated with a single, femtosecond laser pulse, which is perpendicularly incident onto the substrate plane. The intensity of the pulse is adjusted to a value sufficiently low that the parts of the substrate far away from the particles are not affected. Nevertheless, the substrate surface under and near a particle will be ablated if the local intensity enhancement in the optical near-field is high enough. ${ }^{7}$ Thus, the resulting ablation pattern in the substrate, which can be imaged by scanning electron or atomic force microscopy (AFM), represents a nonlinear "photograph" of the optical near-field intensity distribution of the nanoparticle under study. Because of the short duration of the laser pulse, a smearing out of the resulting structures due to thermal conduction, as it can appear for nanosecond pulses, does not occur.

Our investigations cover optical near-fields of both dielectric and metallic nanostructures. In the case of dielectric nanoparticles, we have used polystyrene spheres, available as monodisperse colloidal suspensions, with different diameters in the range of a few hundred nanometers. These particles were deposited on a silicon substrate (commercial silicon

\footnotetext{
${ }^{\text {a)} E l e c t r o n i c ~ m a i l: ~ p a u l . l e i d e r e r @ u n i-k o n s t a n z . d e ~}$

${ }^{\text {b)} P r e s e n t ~ a d d r e s s: ~ U n i v e r s i t y ~ o f ~ F r e i b u r g, ~ F a k u l t a ̈ t ~ f u ̈ r ~ P h y s i k, ~} 79104$ Freiburg, Germany.
}

wafer) by means of spin coating. Using properly diluted suspensions one can obtain samples in which nearly all the spheres are isolated and distributed statistically across the surface. ${ }^{8}$ These samples were then illuminated with a slightly focused Ti:sapphire laser $(\lambda=800 \mathrm{~nm})$, providing pulses with a duration of $150 \mathrm{fs}$ and energies up to $10 \mathrm{~mJ}$ per pulse.

For isolated particles in free space, the scattered light field is described by Mie theory. ${ }^{9}$ As long as the dielectric spheres are larger than the light wavelength, one can consider such particles as spherical lenses. In terms of geometrical optics, a sphere with radius $R$ and refractive index $n$ $=1.59$ (polystyrene) has a focus at a distance of about $R / 4$ behind the surface of the sphere. Thus, for a sphere resting on a flat substrate, one expects, in this approximation, a nearfield in the substrate plane which is circular and which has a diameter of roughly $R / 2$. Indeed, in previous experiments, we have shown that micrometer-sized colloid spheres on $\mathrm{Si}$ substrates give rise to nearly circular holes of approximately this diameter when irradiated with a femtosecond laser pulse. $^{7}$

When the particle diameter becomes smaller than the light wavelength, however, this simple approach no longer holds, and one has to use treatments based on Mie theory. Figure 1(a) shows a calculation for the intensity distribution below a sphere with $R / \lambda=0.4$ in a plane touching the particle. The polarization of the incident light is along the $y$ axis. The intensity pattern now no longer has circular symmetry, but rather reflects the dipole character of the optical near-field, reminiscent of a Hertzian dipole. It should be

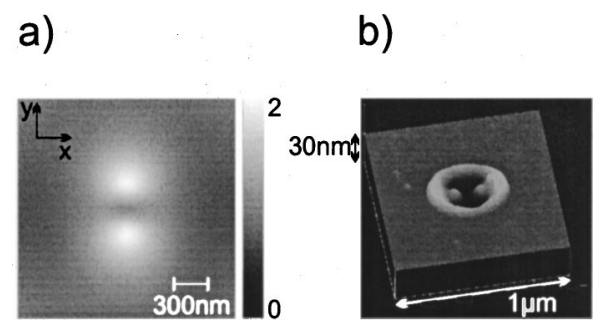

FIG. 1. Near-field of a colloidal sphere $320 \mathrm{~nm}$ in diameter. (a) Left: Mie calculation of the optical near-field intensity in the substrate plane. (b) Right: AFM picture of the silicon substrate under the colloidal sphere after irradiation with a femtosecond laser pulse. The particle itself has been removed due to material ablation. 
a)
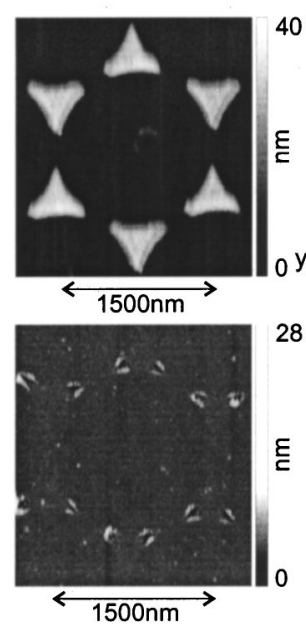

c) b)

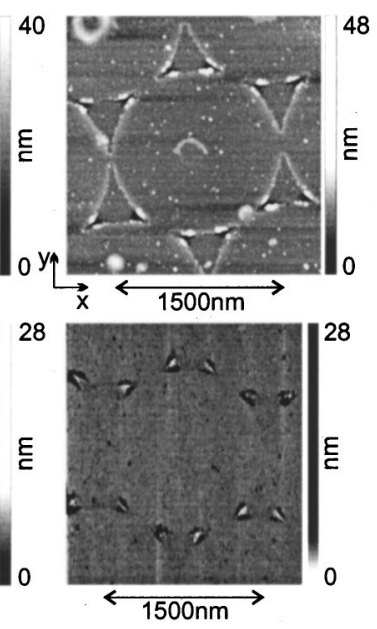

d)

FIG. 2. (a) Top left: gold triangles. (b) Top right: after a 150 fs laser pulse, maximum field enhancement at the tips at the edge perpendicular to the polarization. (c) Bottom left: (b) after chemical removal of the remaining gold. (d) Bottom right: (c) with inverted gray scale; that is, the holes in the $\mathrm{Si}$, corresponding to the areas of high local intensity, appear bright. Polarization of the laser pulse was in the direction of the $y$ axis.

mentioned that in this calculation the particle was taken as an isolated object, that is, the refractive index $n$ of the substrate was assumed to be $n=1$. For a $\mathrm{Si}$ substrate $(n=3.8$ at $\lambda$ $=800 \mathrm{~nm}$ ), a modification of this pattern is to be expected, but the essential features should remain.

The fact that this is indeed the case is shown by the ablation pattern generated by a $320 \mathrm{~nm}$ (PS) sphere [Fig. 1(b)], which corresponds to the situation calculated in Fig. 1(a). The Si surface exhibits a dumbbell-shaped hole structure, with a depth of $10 \mathrm{~nm}$ and a separation of the minima of about $150 \mathrm{~nm}$. The polarization of the incident light was along the dumbbell axis. Around the ablated area, there is an elevated rim, as has already been observed for the larger structures with circular holes. ${ }^{7}$ The composition of this rim has not yet been identified, but we suppose it to consist of $\mathrm{SiO}_{x}$ as an ablation product of the Si substrate.

The similarity between the intensity distribution in Fig. 1(a) and the ablation pattern Fig. 1(b) is obvious. This demonstrates that this method can be used, at least qualitatively, to obtain experimental information about the optical nearfield intensity distribution of a nanoscopic object on a surface.

As already mentioned, in addition to these nonabsorbing particles we have also investigated the optical properties of metallic nanostructures, which were also more complex in shape than the colloidal spheres. One example of such structures are metallic triangles, as they can be obtained, for example, by means of colloid lithography. ${ }^{10,11}$ Using this technique, first, a self-assembled monolayer of polystyrene spheres was grown on a silicon substrate. A thin gold film was then evaporated, and the colloid particles were removed, leaving behind a regular array of gold triangles, as shown in Fig. 2(a).

In addition, these structures were illuminated with a laser pulse of $150 \mathrm{fs}$ duration, and the resulting ablation patterns were analyzed with an AFM. Results for triangles with

a)

b)

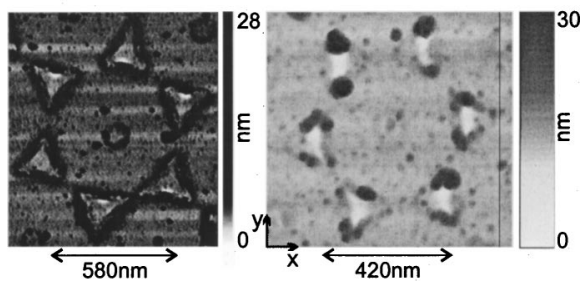

FIG. 3. AFM pictures [inverted gray scale as in Fig. 2(d)] of the near-fields of gold triangles illuminated with a femtosecond laser pulse (polarized along the $y$ axis). (a) Left: mask with $580 \mathrm{~nm}$ colloids, maximum field enhancement in the middle of the edge perpendicular to the polarization. (b) Right: mask with $420 \mathrm{~nm}$ colloids, highest field enhancement for the tips in field direction. In both cases, fragments of the ablated gold triangles have not been removed.

a side length of $450 \mathrm{~nm}$ (produced by a mask with a $1500 \mathrm{~nm}$ sphere diameter) and $25 \mathrm{~nm}$ thickness are shown in Fig. 2(b). The polarization of the incident laser light was again along the $y$ axis. As seen in the figure, most of the gold is removed by the laser pulse, and there are holes, again surrounded by elevated rims, at two corners of each triangle. After chemical removal of the nanoscopic gold droplets, which are scattered over the sample surface as a result of the ablation process, the hole-rim structure becomes more evident [Fig. 2(c)]. We found that the radius of curvature at the tip of the holes can be as small as $5 \mathrm{~nm}$, which was also the tip radius of the original gold triangles. In order to highlight the areas with field enhancement, we present in Fig. 2(d) the same data as in Fig. 2(c) in an inverted gray scale; that is, there, the holes in the $\mathrm{Si}$, corresponding to the areas of high local intensity, appear bright.

At this point we should mention that although we had expected the near-field pattern of a metallic triangle, when irradiated with polarized light, to be anisotropic and display strong field enhancement at the triangle tips, we were still surprised by the orientation of the field enhancement. Taking the triangle as a resonator with a side length of about $\lambda / 2$, one could naively imagine that the field enhancement should develop as in the case of a dipole antenna; that is, at the tips oriented parallel to the polarization of the electric field. As Fig. 2(c) shows, however, this is not the case. Instead, the intensity is largest for the tips aligned along the direction perpendicular to the electric field, and the tip pointing in the direction of the field bears no indication of field enhancement.

This result is at variance, at first sight, with the studies by Crozier et al., ${ }^{3}$ where the field distribution of triangular gold structures, located in regular arrays on a silicon substrate, has been calculated for irradiation with infrared light. In these calculations, which have also been supported by far-field absorption measurements, the field enhancement always was largest at the tip pointing in the direction of the electric field. A detailed comparison between these results and our investigations is hampered by the fact that Crozier et al. consider irradiation from the substrate side where the wavelength of the electromagnetic radiation is considerably reduced and also by the distinctly lower frequencies used by the authors.

Figure 3 demonstrates that the geometrical dimensions of the antenna in relation to the optical wavelength are of crucial importance, as expected. In the figure we show the 
results for gold triangles, taken under similar conditions as in Fig. 2, but this time for smaller structures (side length 240 and $160 \mathrm{~nm}$ ). For the smallest triangles, the field enhancement is highest for the tip in the field direction, now in agreement with the calculations of Ref. 3. For the intermediate triangle size, however, the field enhancement reaches its maximum not at a tip, but rather in the middle of the edge that is perpendicular to the plane of polarization. These examples show that the plasma resonances in metallic structures can be quite complex. It should be mentioned that similar resonances, although somewhat more blurred, can also be observed in far-field measurements using a confocal scanning microscope. ${ }^{12}$ This technique is not suitable, however, for sizes distinctly smaller than the light wavelength. The results presented here are only some of the first examples for the ablation method, which should now be complemented by more detailed and systematic studies, for example, regarding size, thickness, and material of the nanostructures, as well as the near-field interaction of particle arrays. ${ }^{13}$ The influence of the substrate on the near-field intensity distribution can be investigated by using substrates with different refractive indices (bulk glass and polymer and metal films have already been tested successfully, the only requirement being that the surfaces have to be sufficiently flat that the modification in topography due to the optical near-field can be distinguished). For a more quantitative analysis of the ablation patterns, one will have to take the different coupling of normal and tangential field components into account. An improved understanding of the ablation processes induced by femtosecond pulses, which is still incomplete, ${ }^{14}$ will also be required. On the other hand, the very local material removal taking place in the near-field of nanostructures might help to shed some light on the ablation mechanisms themselves.

The results presented here are of great interest for the nanostructuring of substrates and for the mapping of field distributions of plasmon resonances with visible and near-IR wavelengths. With infrared spectroscopy $(\lambda=10 \mu \mathrm{m})$, a spatial resolution of $<\lambda / 100$ is possible. ${ }^{15}$ As we have shown, with our technique one is able to produce nanostructuresusing a wavelength in the near-infrared-with lateral dimensions well below the diffraction limit, and one can image details of the optical near-fields also with a resolution better than $\lambda / 100$.

We gratefully acknowledge helpful discussions with $\mathrm{M}$. Bertsch, H.-J. Münzer, F. Lang, J. Graf, and R. Wannemacher. This work was supported by the Deutsche Forschungsgemeinschaft (SFB 513) and the Kompetenznetz Funktionelle Nanostrukturen Baden-Württemberg.

${ }^{1}$ R. Hillenbrand, S. Senz, W. Herget, and U. Gösele, J. Appl. Phys. 94, 2758 (2003)

${ }^{2}$ S. A. Maier, P. G. Kik, and H. A. Atwater, Appl. Phys. Lett. 81, 1714 (2002).

${ }^{3}$ K. B. Crozier, A. Sundaramurthy, G. S. Kino, and C. F. Quate, J. Appl. Phys. 94, 4632 (2003).

${ }^{4}$ D. W. Pohl, W. Denk, and M. Lanz, Appl. Phys. Lett. 44, 651 (1984).

${ }^{5}$ H.-J. Maas, J. Heimel, H. Fuchs, U. C. Fischer, J. C. Weeber, and A. Dereux, J. Microsc. 209, 241 (2003).

${ }^{6}$ R. Hillenbrand, F. Keilmann, P. Hanarp, P. S. Sutherland, and J. Aizpura, Appl. Phys. Lett. 83, 368 (2003).

${ }^{7}$ H.-J. Münzer, M. Mosbacher, M. Bertsch, J. Zimmermann, P. Leiderer, and J. Boneberg, J. Microsc. 202, 129 (2001).

${ }^{8}$ M. Mosbacher, V. Dobler, J. Boneberg, and P. Leiderer, Appl. Phys. A: Mater. Sci. Process. 70, 669 (2000).

${ }^{9}$ G. Mie, Ann. Phys. (Leipzig) 4, 377 (1908).

${ }^{10}$ U. C. Fischer and H. P. Zingsheim, J. Vac. Sci. Technol. 19, 881 (1981).

${ }^{11}$ F. Burmeister, C. Schäfle, T. Matthes, M. Böhmisch, J. Boneberg, and P. Leiderer, Langmuir 13, 2983 (1997).

${ }^{12}$ M. Goncalves and O. Marti, New J. Phys. 5, 160 (2003).

${ }^{13}$ W. Rechberger, A. Hohenau, A. Leitner, J. R. Krenn, B. Lamprecht, and F. R. Aussenegg, Opt. Commun. 220, 137 (2003).

${ }^{14}$ A. V. Bulgakov, I. Ozerov, and W. Marine, Appl. Phys. A: Mater. Sci. Process. 79, 1591 (2004).

${ }^{15}$ R. Hillenbrand, T. Taubner, and F. Keilmann, Nature (London) 418, 159 (2002). 